\title{
TÉCNICA DOS QUANTIS PARA CARACTERIZAÇÃO DE ANOS SECOS E CHUVOSOS (1980-2009): BAIXO CURSO DO APODI-MOSSORÓ/RN
}

\author{
Jander Barbosa Monteiro ${ }^{1}$ \\ Alexsandra Bezerra da Rocha ${ }^{2}$ \\ Maria Elisa Zanella ${ }^{3}$
}

\begin{abstract}
Resumo: $O$ presente trabalho tem por objetivo utilizar a técnica dos quantis para caracterização de períodos secos e chuvosos, correspondente à série histórica no período de 1980 a 2009 da Empresa de Pesquisa Agropecuária do Rio Grande do Norte - EMPARN, por meio da metodologia de Pinkayan e Xavier. Até o presente (2011) e de acordo com as pesquisas em livros e periódicos, a técnica quantílica ainda não havia sido aplicada na área em estudo. Para análise da técnica dos quantis utilizou-se cinco classes: Muito Seco, Seco, Normal, Chuvoso e Muito Chuvoso, em escala anual, auxiliares na sistematização de dados e na obtenção dos valores normais ou habituais para a chuva no baixo curso do rio ApodiMossoró, representadas pelos quantis $Q(0,15), Q(0,35), Q(0,50), Q(0,65)$ e $Q(0,85)$. Obteve-se importantes informações sobre o regime climático da área em estudo, correlacionado às classes extremas (Muito Seco e Muito Chuvoso) servindo de base para estudos sobre impactos socioambientais associados a desastres naturais, bem como, no planejamento dos recursos hídricos, nos estudos hidrológicos, no planejamento urbano e no planejamento agrícola, e possível subsídio para outros trabalhos e projetos de pesquisa.
\end{abstract}

Palavras-Chave: Apodi-Mossoró; Quantis; Eventos extremos.

\section{TECHNICAL CHARACTERISTICS OF THE AMOUNT FOR PERIODS DRY AND WET (1980-2009) FOR THE DOWN STROKE APODI-MOSSORÓ/RN}

\begin{abstract}
The present paper aims at using the Quantis Technique for the determination of drought and rain periods, corresponding to the historical series in the 30-year stretch between 1980-2009 of the Empresa de Pesquisa Agropecuária do Rio Grande do NorteEMPARN, using the Pinkayan and Terezinha Xavier methodology. Up to the present time (2011) according to research in books and periodicals, a quantilic technique has not been applied to this area of investigation. Five categories have been used to analise the quantis technique: very dry, dry, normal, rainy and very rainy. This classification has been set in an annual scale in order to help in data systematization and in the collection of normal or regular rain figures in the low Apodi-Mossoró watercourse, represented by the quantis $Q(0.15), Q(0.35), Q(0.50), Q(0.65)$ e $Q(0.85)$. Important information has been gathered about the climate regime in the area under study correlated to the extreme classes (very dry and very rainy), and these correlations have served as the starting point of research on socioenvironmental impacts associated to natural disasters, as well as in water resources management, hydrology studies, urban and agricultural planning and as a likely source of information for other works and research projects.
\end{abstract}

Key-Words: Apodi-Mossoró; Quantis; Extreme occurrences.

\footnotetext{
${ }^{1}$ Graduação em Geografia, Mestre em Geografia pela Universidade Estadual do Ceará - Fortaleza/CE - E-mail: jander_bm@hotmail.com

${ }^{2}$ Graduação em Geografia, Especialização em Desenvolvimento Regional e Gestão do Território, Mestre em Geografia, Doutoranda do Programa de Pós-Graduação em Geografia - Universidade Federal do Ceará - Fortaleza/CE - E-mail: rbalexsandra@yahoo.com.br

${ }^{3}$ Graduação em Geografia, Mestrado em Organização do Espaço, Doutorado em Meio Ambiente e Desenvolvimento, Professora do Curso de Graduação em Geografia e do Programa de Pós Graduação em Geografia - Universidade Federal do Ceará - E-mail: elizazv@terra.com.br

DOI: 10.7154/RDG.2012.0023.0010
} 


\section{INTRODUÇÃO}

Os eventos extremos de precipitação, em relação aos valores médios, influenciam, tanto direta, quanto indiretamente, a sociedade.

As relações entre as anomalias positivas e negativas das chuvas resultam em impactos que provocam muitos problemas para a cidade e o campo. As populações urbanas, geralmente são mais atingidas quando as chuvas apresentam anomalias positivas, enquanto que as rurais quando as chuvas são escassas.

De acordo com Amorim e Monteiro (2010), aos olhos dos cidadãos e dos decisores, os estados de tempo, caracterizados por uma grande quantidade, intensidade e frequência de precipitação, são na maioria das vezes os responsáveis por calamidades e pela desorganização do ambiente urbano quando acontecem. De maneira geral, a sociedade prefere vivificar os elementos climáticos - neste caso, a chuva - e atribuir-lhes a responsabilidade pelos prejuízos. A opção por uma ocupação do solo, de acordo com as suas necessidades e modelo de vida e, muitas vezes, desconsiderando as vulnerabilidades dos lugares que escolhe para se instalar, geralmente não é alvo de sua análise.

Em meio a tais considerações, tem-se vivenciado nas últimas décadas, em várias regiões de nosso país, que precipitações mais intensas têm provocado inúmeros impactos negativos, com elevado número de mortes, feridos e perdas materiais, principalmente em áreas cujas vulnerabilidades ambientais são mais elevadas.

Os impactos gerados por episódios de chuvas extremas podem, na maioria das vezes, ser enquadrados na categoria de desastres naturais, dependendo de sua magnitude e extensão espacial (BRANDÃO, 2001). Os decretos de emergência adotados com muita frequencia em vários municipios das diversas regiões brasileiras configuram essa situação.

Os desastres naturais mais frequentes no Brasil são as inundações urbanas, os deslizamentos de terra, as secas e a erosão, todos relacionados ao sistema climático. Assim, os estudos relacionados às secas e aos impactos das chuvas ganharam uma maior repercussão no território brasileiro, sendo analisados em diversas áreas do conhecimento, como a geografia, a meteorologia, a sociologia, a engenharia sanitária, dentre outras áreas.

No Estado do Rio Grande do Norte, observa-se com maior freqüência, a ocorrência de dois tipos desses desastres: as secas e as inundações urbanas. Ambos estão relacionados às chuvas (seja pelo excesso ou falta destas) e, nesse contexto, percebe-se a importância de se 
analisar os desastres naturais desencadeados pela baixa pluviosidade ou pelo excesso de chuvas, os riscos a que estão sujeitas as populações que habitam áreas consideradas vulneráveis, e até mesmo observar como tais desastres afetam de forma diferenciada as áreas urbanas e rurais.

Porém, há uma grande dificuldade entre os pesquisadores em determinar valores extremos. Afinal, como estabelecer valores (em milímetros) confiáveis para regiões com características climáticas diferenciadas no país e a partir de que totais há a configuração de desastres?

Geralmente, a sociedade não consegue enxergar tal limite, acreditando que os danos sempre são culpa de uma exceção, algo que acontece ao acaso, proveniente de um agente externo, originado para além dos parâmetros normais, como se normalidade e desastre se transformassem em dois mundos separados por uma linha mágica (CRUZ, 2003).

Não se pode esquecer que em algumas cidades brasileiras as chuvas podem ser abundantes e nesse sentido, registram-se valores normais relativamente elevados. Porém, em outras regiões brasileiras, como em algumas cidades da região Nordeste, o habitual é chover de forma mal distribuída e às vezes até escassa.

Nesse sentido, esta pesquisa objetiva estabelecer e discutir valores normais e extremos para a chuva na região do baixo curso do rio Apodi-Mossoró/RN, através da utilização da técnica estatística dos quantis, em uma série histórica de 30 anos (1980-2009). Esta técnica permite uma análise confiável para estabelecer valores normais e extremos, conforme metodologia apresentada neste trabalho.

Para atingir tais objetivos, realizou-se uma pesquisa documental por meio de consultas a informações qualificadas presentes em órgãos relacionados à temática de estudo (Empresa de Pesquisa Agropecuária do Rio Grande do Norte - EMPARN) e utilização de técnicas que permitem caracterizar, de forma mais confiável, um determinado evento como extremo (técnica dos quantis).

O desenvolvimento de estudos sobre a influência e os impactos associados a valores extremos, como também de metodologias com o intuito de entender os desastres naturais, torna-se fundamental, de forma que esses estudos sirvam como subsídios para a gestão pública no tocante à tomada de decisões que possibilitem medidas preventivas para mitigar os impactos deles provenientes. 


\section{Caracterização da Área de Estudo}

O baixo curso do rio Apodi-Mossoró esta localizado entre os paralelos 4 57' 12" e 5o 08' '5" de latitude sul e os meridianos $37^{\circ} 02^{\prime} 12^{\prime}$ e $37^{\circ} 20^{\prime} 2^{\prime \prime}$ de longitude oeste (Mapa 1), cuja extensão territorial é de $973.703 \mathrm{Km}^{2}$. Abrange a zona rural do município de Mossoró (49\%), na porção noroeste mageada pela BR 304 e BR110, parte do município de Areia Branca acessado pela BR 110, estendendo-se até o litoral setentrional (22\%), parte do município de Grossos alcançada pelas RN 012 e RN 013 estendendo-se até litoral (8\%) e a zona rural do município de Serra do Mel ladeada pela BR 110 (21\%).

No tocante a geodiversidade trata-se de uma área caracterizada pela presença de uma chapada, de tabuleiros costeiros, de planície flúvio-marinha, de faixa costeira com praias, falésias e dunas, apresentando um mosaico de solos e diferentes conjuntos vegetacionais.

O regime térmico da região é caracterizado, basicamente, por temperaturas elevadas e amplitudes térmicas reduzidas. A temperatura média anual gira em torno de $27^{\circ} \mathrm{C}$, onde julho representa o mês mais frio, com média de 26,5으. As temperaturas máximas e

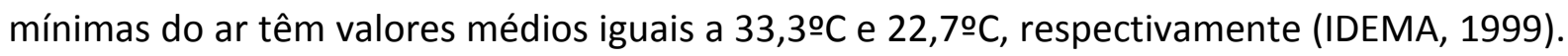
Isto está relacionado à baixa latitude e à ausência de fatores geográficos influenciadores. Os ventos predominantes na região são de E-SE e tem velocidade média anual de 4,0 m/s.

Poucos são os estudos climatológicos realizados nesta área. Alguns trabalhos podem ser citados como os de Carmo Filho et al. (1991), Borges (1983), Aguiar (1988), Baptista et al. (2005), Rocha (2010), dentre outros. Tais resultados foram importantes para o estudo aqui apresentado. O estudo sobre balanço hídrico (1970-2009) realizado por Rocha (2011) indicou que a área drenada pelo baixo curso do rio Apodi-Mossoró é semiárida, e que não é apenas a falta de chuvas a responsável pela oferta insuficiente de água na região, mas a sua má distribuição, associada à alta taxa de evapotranspiração, agravada pelas secas periódicas, e pela diminuição das chuvas do interior em relação ao litoral.

Segundo Rocha (2011), o tipo climático da área em estudo potencializa o desenvolvimento de determinadas atividades econômicas, que por sua vez impulsiona a dinâmica socioeconômica da região. Dentre estas atividades destaca-se a produção de sal, que tem demanda de altas temperaturas, baixo teor de umidade, elevada evaporação, baixas precipitações, intensa radiação solar, ventos constantes e de maior velocidade, relevo plano e solo impermeável. 


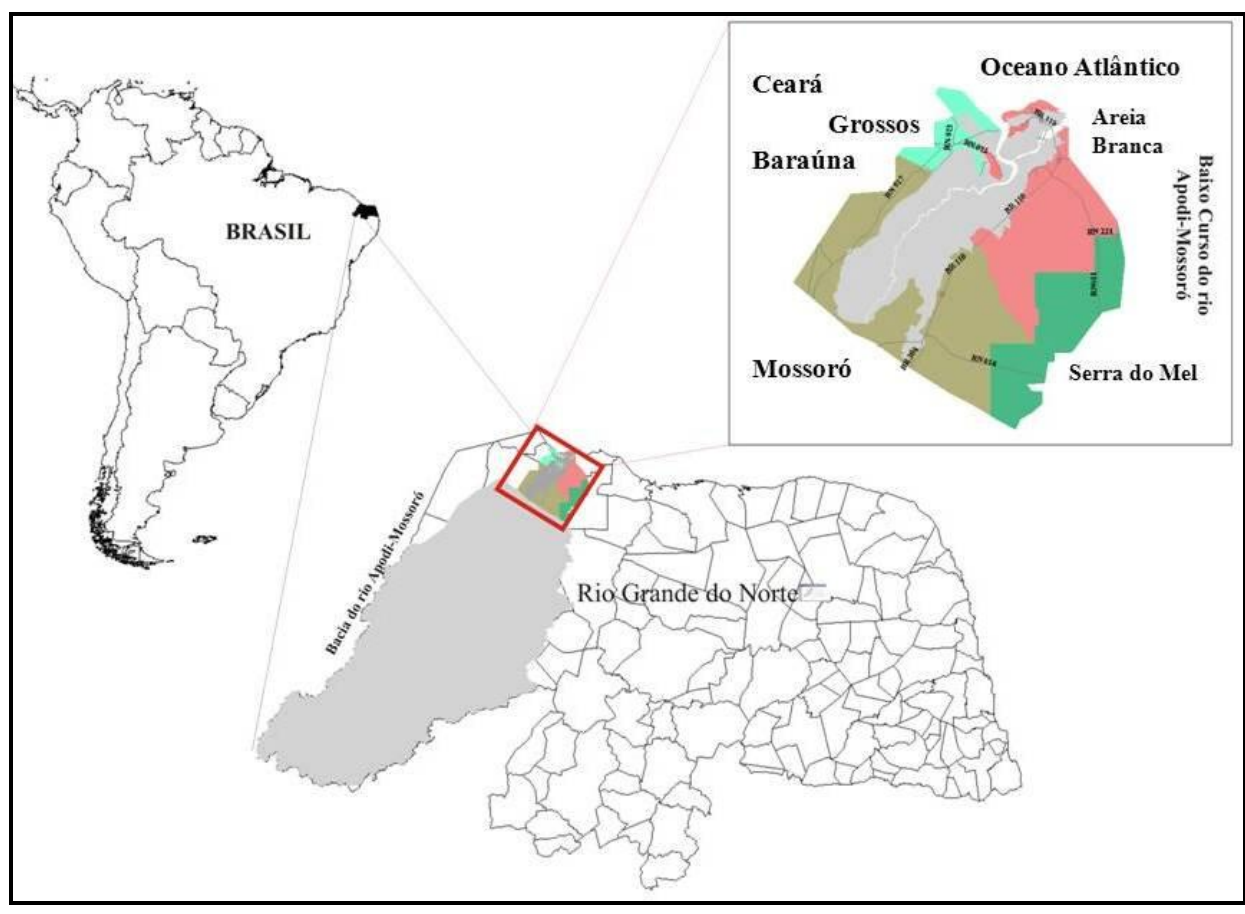

Mapa 1: Localização do baixo curso do rio Apodi-Mossoró-RN. Fonte: Rocha, A.B (2011)

É importante ressaltar que a área em estudo apresenta os três critérios estabelecidos pelo Grupo de Trabalho Interministerial (GTI, 2004) responsável pela nova delimitação das regiões semiáridas, ou seja, precipitação pluviométrica média anual inferior a $800 \mathrm{~mm}$, índice de aridez de até 0,5 e risco de seca maior que $60 \%$.

\section{METODOLOGIA}

Para a contemplação do objetivo proposto neste trabalho, foi analisado o comportamento da chuva em uma série histórica de 30 anos (1980-2009), por meio das informações qualificadas presentes na Empresa de Pesquisa Agropecuária do Rio Grande do Norte EMPARN. A partir destas informações, aplicou-se a técnica estatística dos quantis, de acordo com a metodologia de Pinkayan (1966) e Xavier (2001, 2004, 2007).

Para a aplicação da técnica, levaram-se em consideração apenas as informações qualificadas contidas em três postos dos quatro municípios inseridos no baixo curso: Mossoró (lat: 5011'15”S long:370 20 '39”W, alt 115), Grossos (lat: 40 58'47”W long: 370 09' 17' S alt 104) e Areia Branca (lat 40 57' 22”S long: 37ㅇ 08' 13“'W alt 5). Isto ocorreu porque os dados do posto pluviométrico da EMPARN referente ao município de Serra do Mel estão incompletos, apresentando apenas informações a partir de 1993, sendo que nos anos de 
1995, 1996, 1997, 2000, 2001, 2002, 2005 e 2006 não constam dados, sendo impossível incluí-los nesta análise.

Para o entendimento da utilização da técnica estatística neste trabalho e a noção de quantil, supõe-se que a chuva em um determinado local, acumulada em certo intervalo (mês, bimestre, trimestre, quadrimestre, semestre, ou até mesmo o ano inteiro etc.), com respeito a anos consecutivos, possa ser representada em termos por uma variável aleatória contínua. Dizer que a chuva é uma variável aleatória significa que o valor da sua altura acumulada (em milímetros) não poderá ser previsto com uma exatidão determinística, mas na verdade ela vai ser de natureza probabilística. Ou seja, pode-se atribuir uma probabilidade para que a altura da chuva fique compreendida entre dois limites arbitrariamente escolhidos.

Desta forma, os totais de chuva acumulada em cada mês de cada ano da série histórica são agrupados em uma única tabela e, em seguida, os valores são somados para obtenção do total pluviométrico acumulado durante cada ano. Posteriormente, os valores anuais são ordenados (do menor para o maior), no intuito de aplicar a técnica estatística (Figura 2).

Com os valores ordenados em uma única tabela, pode-se aplicar a técnica e estabelecer os valores dos quantis (Figura 2). Os quantis são as medidas de separação para as distribuições da amostra. Um quantil de ordem p é um valor numérico que secciona a distribuição em duas partes permitindo separar uma amostra em duas massas de observações numéricas, com 100xp\% dos elementos localizados à esquerda do quantil "amostral" e os demais $100 x(1-p) \%$, à direita. A escolha dos quantis a serem calculados é realizada pelo próprio pesquisador, podendo variar de acordo com o objetivo da pesquisa.

Por exemplo, se a intenção é delimitar valores extremos de chuva para a região estudada, pode-se utilizar o quantil de ordem $5 \%$ (valores extremos inferiores para chuva acumulada em determinado ano) e o quantil de ordem 95\% (valores extremos superiores para a chuva acumulada em determinado ano). De forma simples, isso significa que, de acordo com os totais acumulados em cada ano da série histórica (30 anos) e que foram ordenados do menor valor para o maior valor, $5 \%$ destes valores estarão abaixo do $Q(0,05)$, ou seja, representarão valores extremos de chuva (baixa pluviosidade).

No entanto, no outro extremo da tabela, os valores acima do $Q(0,95)$ representam os valores extremos superiores de chuva (elevada pluviosidade). Assim, pode-se concluir que, neste caso, apenas em 1 ou 2 anos da série estudada, pode-se obter valores extremos de 
chuva acumulada no ano, tanto para baixa pluviosidade (anos extremamente secos), como para elevada pluviosidade (anos extremamente chuvosos).

Para o presente trabalho, não utilizaram-se estes quantis extremos. Foi realizada a mesma divisão que Pinkayan (1966) utilizou em seu trabalho, uma divisão em 5 classes: Muito Seco, Seco, Normal, Chuvoso e Muito Chuvoso. Estas são representadas pelos seus respectivos quantis $Q(0,15), Q(0,35), Q(0,50), Q(0,65)$ e $Q(0,85)$.

As classes extremas (Muito Seco e Muito Chuvoso), dessa forma, apresentam um intervalo relativamente menor (apenas 15\%), o que permite um tratamento matemático mais coerente e confiável que leva em consideração uma distribuição normal, enquanto que a classe Normal apresenta o maior intervalo (30\%). No intuito de facilitar a compreensão da distribuição normal utilizando o quantil para os anos estudados na série histórica, pode-se observar o esquema abaixo (figura 1), o qual ordena os valores acumulados de chuva do ano mais seco para o ano mais chuvoso da série.

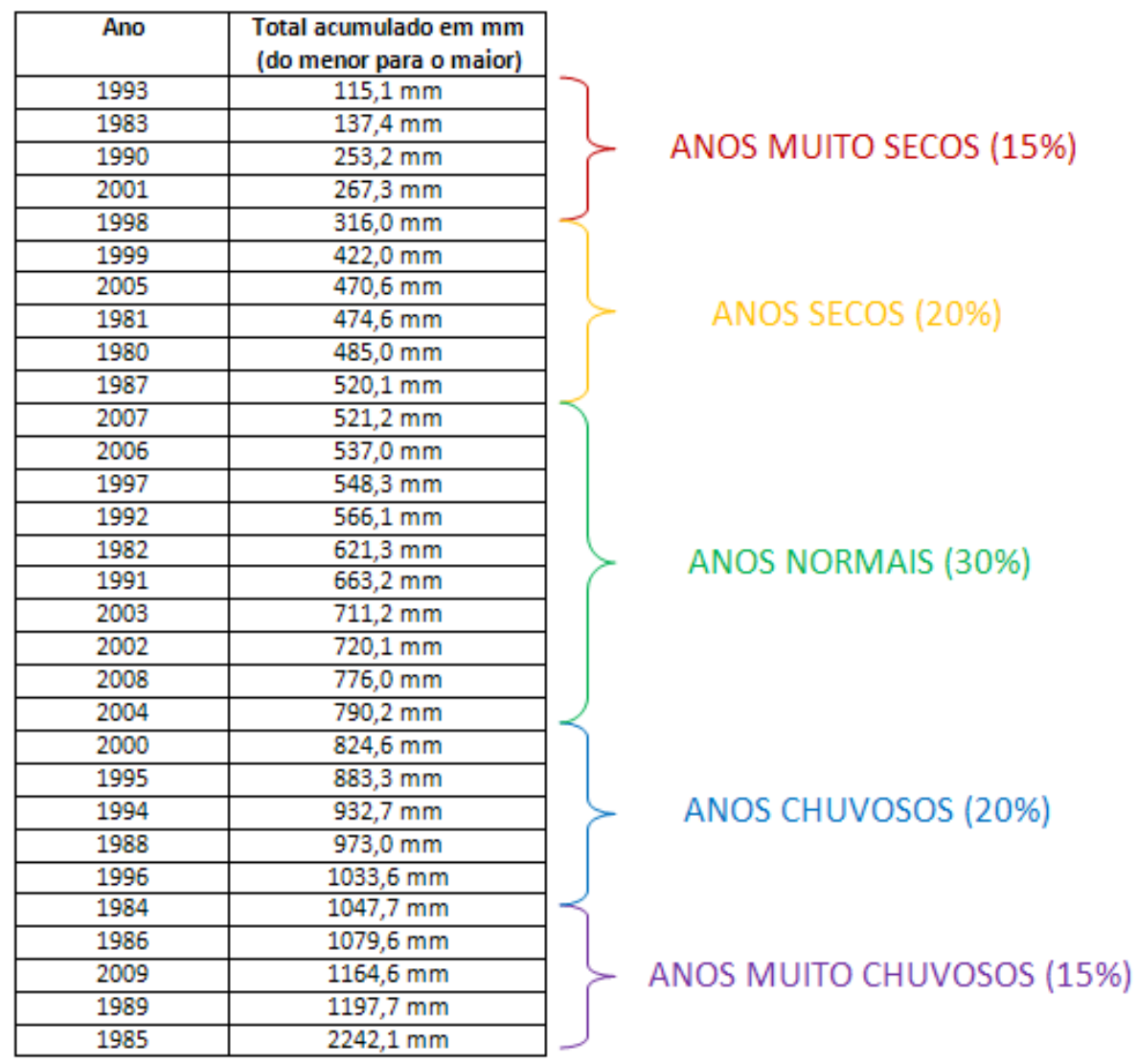

Figura 1 - Distribuição dos valores de chuva acumulada nos anos da série histórica e suas possíveis classificações de acordo com a divisão proposta na pesquisa

A figura acima exemplifica, de forma prática, uma possível classificação para os anos. Porém, para estabelecer de forma precisa os intervalos em $\mathrm{mm}$ para cada quantil e a respectiva 
classificação dos anos, faz-se necessário estabelecer os quantis $Q(0,15), Q(0,35), Q(0,50)$, $Q(0,65)$ e $Q(0,85)$, de acordo com as informações qualificadas presentes nos postos pluviométricos utilizados, as quais serão analisadas para compor a tabela e realização dos cálculos estatísticos.

Estudo realizado por Monteiro (2011) no Estado do Ceará utilizando a técnica quantílica, revelou que os valores normais e extremos para a chuva variam em determinadas regiões do Estado, sendo necessária a utilização de informações qualificadas de postos pluviométricos de municípios próximos para a realização do cálculo estatístico de forma mais confiável, considerando algumas regiões pluviométricas homogêneas. Outros autores também foram importantes para discutir o tema, como: Zanella (2006), Beniston (2004), Castilho (2005), Brandão (2001), Cruz (2003).

Nesse sentido, também foi levado em consideração nesta pesquisa a pluviosidade registrada nos três postos selecionados e na série histórica analisada (1980-2009), no intuito de analisar e ratificar se tais valores de chuva (dos postos selecionados) podem ser considerados como homogêneos (sem bruscas variações de posto para posto).

Uma interpretação simples e todos os passos da metodologia para aplicação da técnica pode ser visualizado na figura 2 .

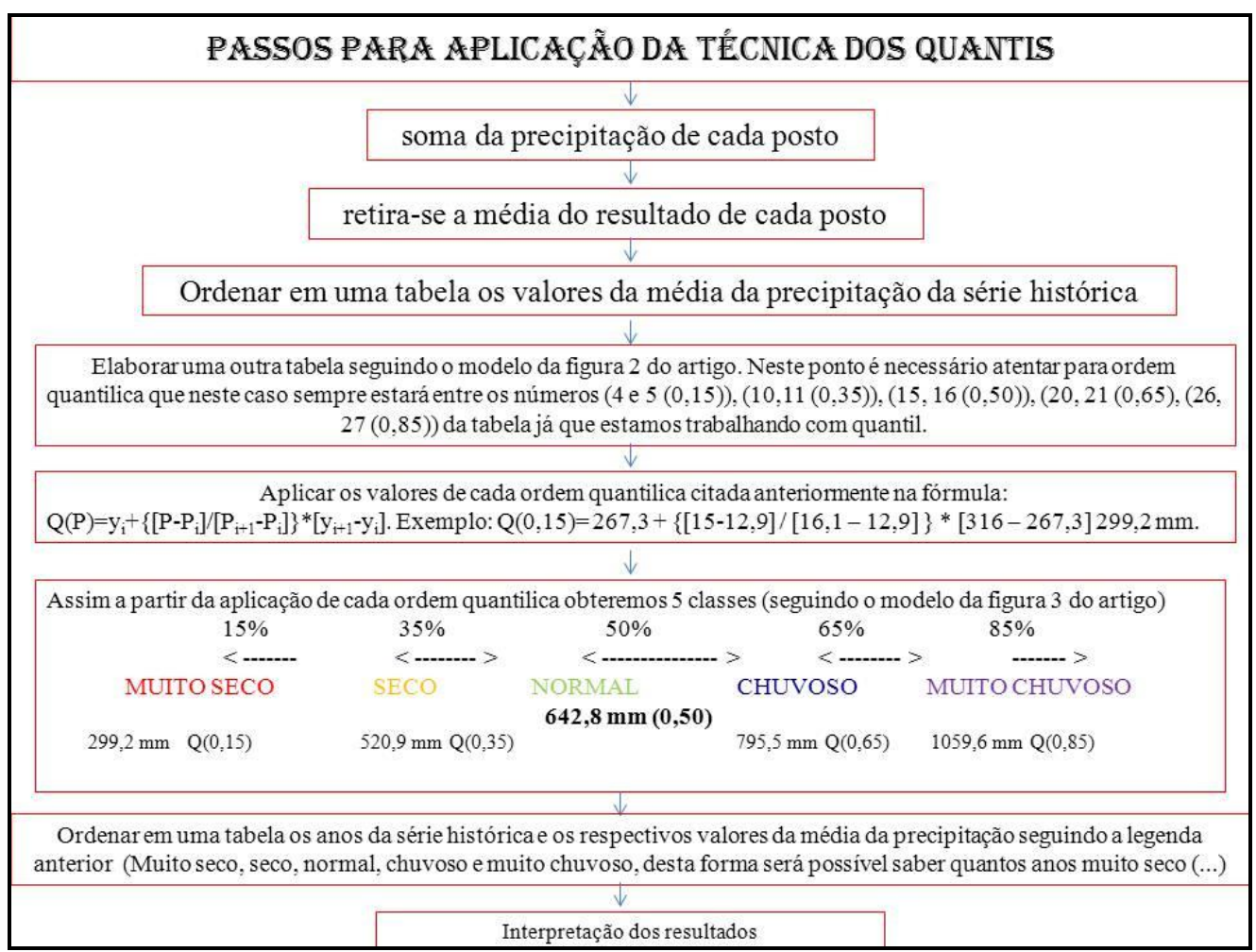

Figura 2 - Passos da metodologia dos quantis para estudos climatológicos, com foco em eventos extremos e desastres naturais. Fonte: Monteiro (2011). 


\section{RESULTADOS E DISCUSSÕES}

O clima impôs a esse território, sem dúvida, algumas restrições. Grande parte do Estado enquadra-se no clima semiárido, caracterizado pela cobertura vegetal pertencente ao domínio da Caatinga, insulada entre a Chapada do Araripe $(800 \mathrm{~m}$ a $1100 \mathrm{~m})$ e o Planalto da Borborema (670m a $1100 \mathrm{~m})$. As secas periódicas assumem a característica de catástrofes em decorrência da densidade demográfica e da dependência da agricultura e da pecuária. As secas cíclicas e de intensidades variáveis têm reflexos na sociedade e no meio natural.

As chuvas se concentram em quatro meses (fevereiro, março, abril e maio). Essa sazonalidade está relacionada aos sistemas atmosféricos e oceânicos que atuam principalmente nas baixas latitudes, às estabilidades atmosféricas no período de inverno e primavera, e às instabilidades no período sazonal do verão e do outono, com ocorrência de chuvas concentradas em quatro meses do ano. Analisando-se os indicadores da evaporação, tem-se que a região, caracteriza-se por ter valores médios anuais de evaporação na ordem dos $2.077,6 \mathrm{~mm}$. No tocante a insolação apresenta uma variação anual entre 2.600 a 3.000 horas de sol por ano.

Os valores dos trinta anos (média dos valores acumulados nos postos pluviométricos) foram ordenados de forma crescente, conforme figura 3, para posterior aplicação da técnica dos quantis, identificando os quantis $Q(0,15), Q(0,35), Q(0,50), Q(0,65)$ e $Q(0,85)$.

\begin{tabular}{|c|c|c|c|c|c|c|c|c|c|c|}
\hline i & 1 & 2 & 3. & $\overline{4}$ & 5 & 6 & 7 & $\&$ & 2 & 10 \\
\hline$y(\mathrm{~mm})$ & 115,1 & 137,4 & 253,2 & 267,3 & 316,0 & 422,0 & 470,6 & 474,6 & 485,0 & 520,1 \\
\hline \multirow[b]{2}{*}{$\mathrm{P}=\mathrm{i} /(\mathrm{N}+\mathrm{l})$} & $1 / 31$ & $2 / 31$ & $3 / 31$ & $4 / 31$ & $5 / 31$ & $6 / 31$ & $7 / 31$ & $8 / 31$ & $9 / 31$ & $10 / 31$ \\
\hline & 0.032 & 0.064 & 0.096 & 0.129 & 0.161 & 0.193 & 0.225 & 0.258 & 0.290 & 0.322 \\
\hline $\bar{i}$ & 11 & 12 & 13 & 14 & 15 & 16 & 17 & 18 & 19 & 20 \\
\hline$y(\mathrm{~mm})$ & 521,2 & 537,0 & 548,3 & 566,1 & 621,3 & 663,2 & 711,2 & 720,1 & 776,0 & 790,2 \\
\hline \multirow{2}{*}{$P_{2}=i /(N+1)$} & $11 / 31$ & $12 / 31$ & $13 / 31$ & $14 / 31$ & $15 / 31$ & $16 / 31$ & $17 / 31$ & $18 / 31$ & $19 / 31$ & $20 / 31$ \\
\hline & 0.354 & 0.387 & 0.419 & 0.451 & 0.483 & 0.516 & 0.548 & 0.580 & 0.612 & 0.645 \\
\hline $\bar{i}$ & 21 & 22 & 23 & 24 & 25 & 26 & 27 & 28 & 29 & 30 \\
\hline$y(\mathrm{~mm})$ & 824,6 & 883,3 & 932,7 & 973,0 & 1033,6 & 1047,7 & 1079,6 & 1164,6 & 1197,7 & 2242,1 \\
\hline \multirow{2}{*}{$\mathrm{P}=\mathrm{i} /(\mathrm{N}+\mathrm{l})$} & $21 / 31$ & $22 / 31$ & $23 / 31$ & $24 / 31$ & $25 / 31$ & $26 / 31$ & $27 / 31$ & $28 / 31$ & $29 / 31$ & $30 / 31$ \\
\hline & 0.677 & 0.709 & 0.741 & 0.774 & 0.806 & 0.838 & 0.870 & 0.903 & 0.935 & 0.967 \\
\hline
\end{tabular}

Figura 3 - Valores ordenados para aplicação da técnica dos quantis no baixo curso do rio Apodi-Mossoró. 
Após a utilização da técnica estatística dos quantis, foram obtidos valores em $(\mathrm{mm})$ estimados para os quantis $Q(0,15), Q(0,35), Q(0,50), Q(0,65)$ e $Q(0,85)^{4}$, dispostos nas cinco classes, conforme modelo esquemático abaixo (Figura 4), de forma que 15\% e $85 \%$ representam as classes extremas (muito seco e muito chuvoso).

Pode-se perceber através da figura 4 que a região do baixo curso do rio Apodi-Mossoró está representada por um valor mediano aproximado anual de $642,8 \mathrm{~mm}$, sendo que, os intervalos estabelecidos $(\mathrm{mm})$ para cada classe extrema indicaram que valores abaixo de 299,2 mm serão considerados como muito seco e acima de 1059,6 mm como muito chuvosos. A média da precipitação, a classificação e o comportamento pluviométrico para cada ano analisado podem ser visualizados na tabela 1.

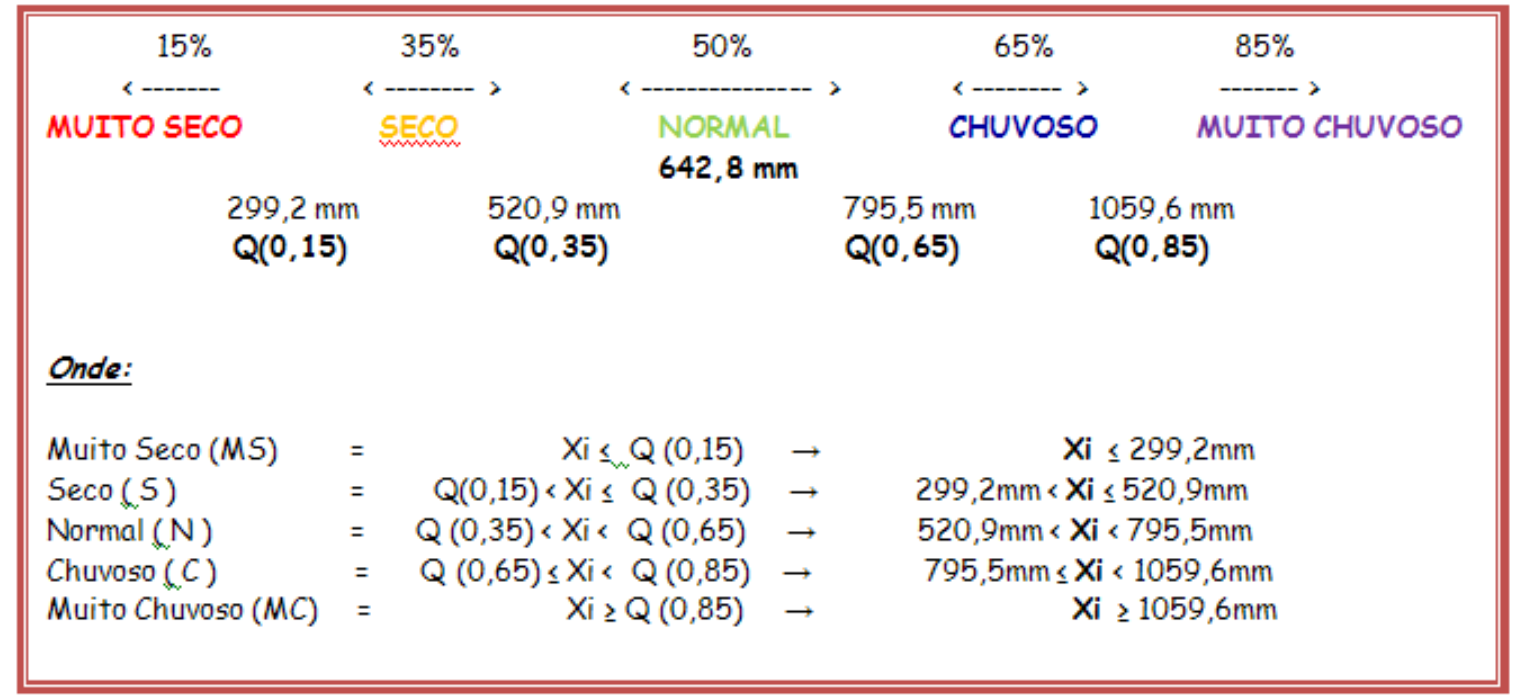

Figura 4 - Valores estabelecidos para os quantis do baixo curso. Fonte: conforme metodologia de Monteiro (2011).

O comportamento pluviométrico com um período chuvoso (de quatro meses) e um período seco (oito meses) ocasionam a mobilização de uma maior quantidade de sedimentos nos períodos secos, pois é nesse período que vão atuar mais intensamente os ventos alísios de leste-sudeste que caracterizam, de maneira geral, a formação e a migração dos campos de dunas e a permanência de areias mais secas nos campos dunares devido às baixas precipitações.

A Zona de Convergência Intertropical - ZCIT é o sistema atmosférico de maior importância no estabelecimento da quadra chuvosa, na área em análise. Fenômeno de grande escala, é uma

\footnotetext{
${ }^{4}$ Onde, $Q(0,15)=267,3+\{[15-12,9] /[16,1-12,9]\} *[316-267,3]=Q(0,15)=299,2 \mathrm{~mm}$

$Q(0,35)=520,1+\{[35-32,2] /[35,4-32,2]\} *[521,1-520,1]=Q(0,35)=520,9 \mathrm{~mm}$

$Q(0,50)=621,3+\{[50-48,3] /[51,6-48,3]\} *[663,2-621,3]=Q(0,50)=642,8 \mathrm{~mm}$

$Q(0,65)=790,2+\{[65-64,5] /[67,7-64,5]\} *[824,6-790,2]=Q(0,65)=795,5 \mathrm{~mm}$

$Q(0,85)=1047,6+\{[85-83,8] /[87,0-83,8]\} *[1079,6-1047,6]=Q(0,85)=1059,6 \mathrm{~mm}$
} 
extensa região de convergência de 3 a 5 graus de largura na faixa de encontro dos alísios de Nordeste e Sudeste. A ZCIT se localiza sobre a isoterma de maior temperatura e é mais intensa sobre o mar.

As informações sobre a dinâmica das chuvas representadas na tabela 1 podem ser comparadas e qualificadas com os dados do Instituto Nacional de Pesquisas Espaciais- INPE, Climate Prediction Center-CPC e do Centro de Previsão de Tempo e Estudos ClimáticosCPTEC no que diz respeito à influência dos sistemas atmosféricos e oceânicos para o baixo curso do rio Apodi-Mossoró referente ao período em análise.

Tabela 1 - Classificação de cada ano da série histórica utilizada (1980-2009), de acordo com os valores estabelecidos para cada classe quantílica.

\begin{tabular}{|c|c|c|}
\hline ANO & MÉDIA (PLUVIOSIDADE) & CLASSIFICAÇÃO \\
\hline 1980 & 485,0 & SECO \\
\hline 1981 & 474,6 & SECO \\
\hline 1982 & 621,3 & NORMAL \\
\hline 1983 & 137,4 & MUITO SECO \\
\hline 1984 & 1047,7 & CHUVOSO \\
\hline 1985 & 2242,1 & MUITO CHUVOSO \\
\hline 1986 & 1079,6 & MUITO CHUVOSO \\
\hline 1987 & 520,1 & SECO \\
\hline 1988 & 973,0 & CHUVOSO \\
\hline 1989 & 1197,7 & MUITO CHUVOSO \\
\hline 1990 & 253,2 & MUITO SECO \\
\hline 1991 & 663,2 & NORMAL \\
\hline 1992 & 566,1 & NORMAL \\
\hline 1993 & 115,1 & MUITO SECO \\
\hline 1994 & 932,7 & CHUVOSO \\
\hline 1995 & 883,3 & CHUVOSO \\
\hline 1996 & 1033,6 & CHUVOSO \\
\hline 1997 & 548,3 & NORMAL \\
\hline 1998 & 316,0 & SECO \\
\hline 1999 & 422,0 & SECO \\
\hline 2000 & 824,6 & CHUVOSO \\
\hline 2001 & 267,3 & MUITO SECO \\
\hline 2002 & 720,1 & NORMAL \\
\hline 2003 & 711,2 & NORMAL \\
\hline 2004 & 790,2 & NORMAL \\
\hline 2005 & 470,6 & SECO \\
\hline 2006 & 537,0 & NORMAL \\
\hline 2007 & 521,1 & NORMAL \\
\hline 2008 & 776,9 & NORMAL \\
\hline 2009 & 1164,6 & MUITO CHUVOSO \\
\hline
\end{tabular}

Fonte: dados elaborados para esta pesquisa, a partir da base de dados da EMPARN.

Já o sistema oceânico mais atuante para o período em análise foi o El niño, variando entre fraco (nos anos de 1991, 1994, 2002, 2003, 2005), moderado (nos anos de 1987, 1993, 1995, 2002, 2003) e forte (em 1982, 1983, 1992, 1997, 1998). Existe também a classificação para 
anos neutros (1980, 1981, 1984, 1986, 1990, 2004, 2007), importantíssimos na análise de eventos extremos, verificando-se situações de longas estiagens ou chuvas extremas, mesmo em condições de neutralidade no Pacífico Equatorial. Já a La niña variou de moderada a fraca (para os anos de 1985, 1988, 1989, 1999, 2000, 2001, 2006, 2008, 2009).

Assim, os sistemas atmosféricos e oceânicos influenciam, temporal e espacialmente, a distribuição pluviométrica, auxiliando no conhecimento dos padrões predominantes de precipitação em diferentes escalas e contribuindo, assim, no planejamento dos recursos hídricos, nos estudos hidrológicos, no planejamento regional, urbano e agrícola, entre outros fatores.

O ano de 1985 corresponde ao valor anual extremo de chuvas na série histórica, com um total de $2.242,1 \mathrm{~mm}$, o que corresponde a um desvio positivo em relação ao quantil estabelecido para a classe muito chuvoso $(1059,6 \mathrm{~mm})$ de $1.187 \mathrm{~mm}$, correspondendo a um percentual superior a $100 \%$, registrando diversos impactos negativos na população no Município de Mossoró por ocasião do período chuvoso.

Para exemplificar, o Jornal Gazeta do Oeste, do dia 07 de maio de 1985, registra no município, principalmente na cidade, inundações que desabrigaram parte da população, principalmente aquela localizada às margens do rio Apodi-Mossoró, que cruza a cidade. Comércios foram destruídos e alagados, a população ficou sem água potável e energia, dentre outros transtornos. A figura 5 registra as condições das ruas da cidade de Mossoró naquela data.

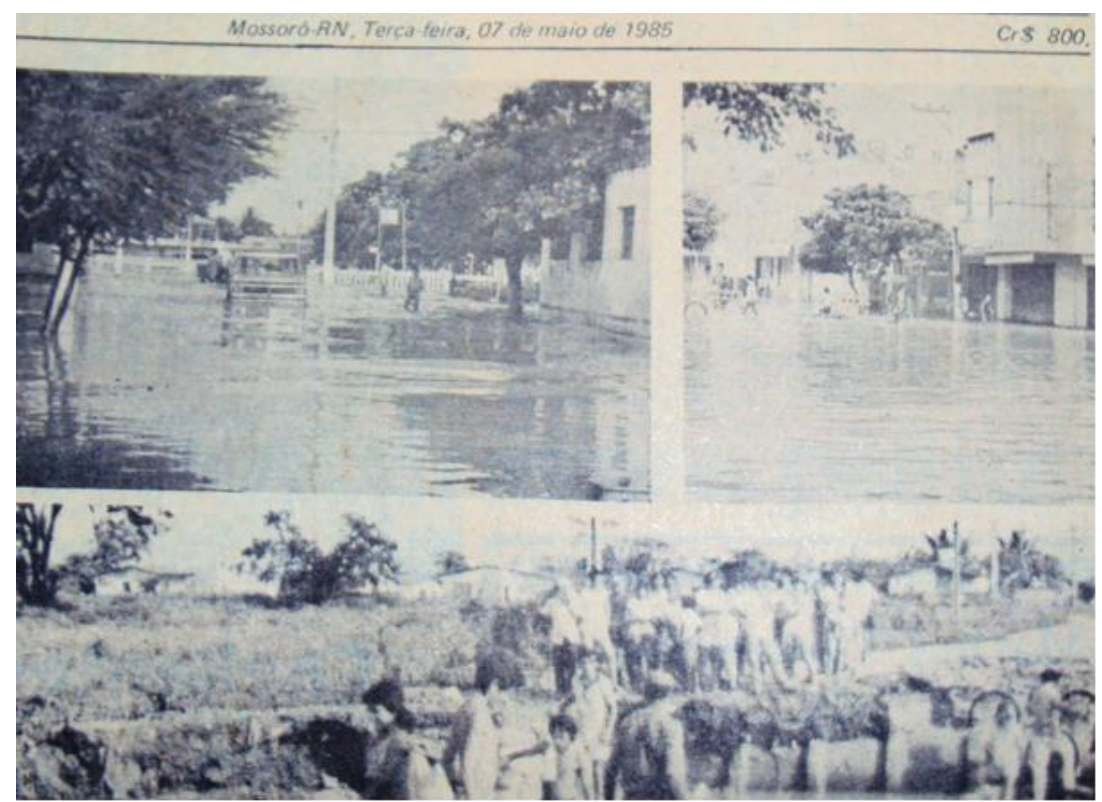

Figura 5 - Impactos das chuvas no município de Mossoró Fonte: Jornal Gazeta do Oeste, 07 de Maio de 1985. 
Por outro lado, o ano de 1983, registrou o valor de 137,4mm, resultando em seca catastrófica para a região, prejudicando principalmente as atividades agropecuárias, além da falta de água para abastecer as atividades produtivas e a população da cidade de Mossoró e cidades menores daquela região.

Por meio do gráfico 1, pode-se perceber que a classificação dos trinta anos da série histórica analisada em cada uma das cinco classes propostas no trabalho, seguem uma distribuição normal praticamente perfeita, ou seja, na classe muito seco representou, aproximadamente de 13 a 14\%, a classificação seco (20\%), a normal ou habitual (cerca de 33\%), chuvosa (20\%) e a muito chuvosa (aproximadamente de $13 \%$ a $14 \%$ ).

Tal análise permite avaliar corretamente o grau de severidade das secas ocorridas no passado, e de inteiro acordo com as cronologias históricas levantadas por Carmo Filho et al (1991). O gráfico 1 apresenta uma curva suave e simétrica, justificando a utilização da técnica e comprovando a coerência do cálculo estatístico na avaliação de valores normais e extremos.

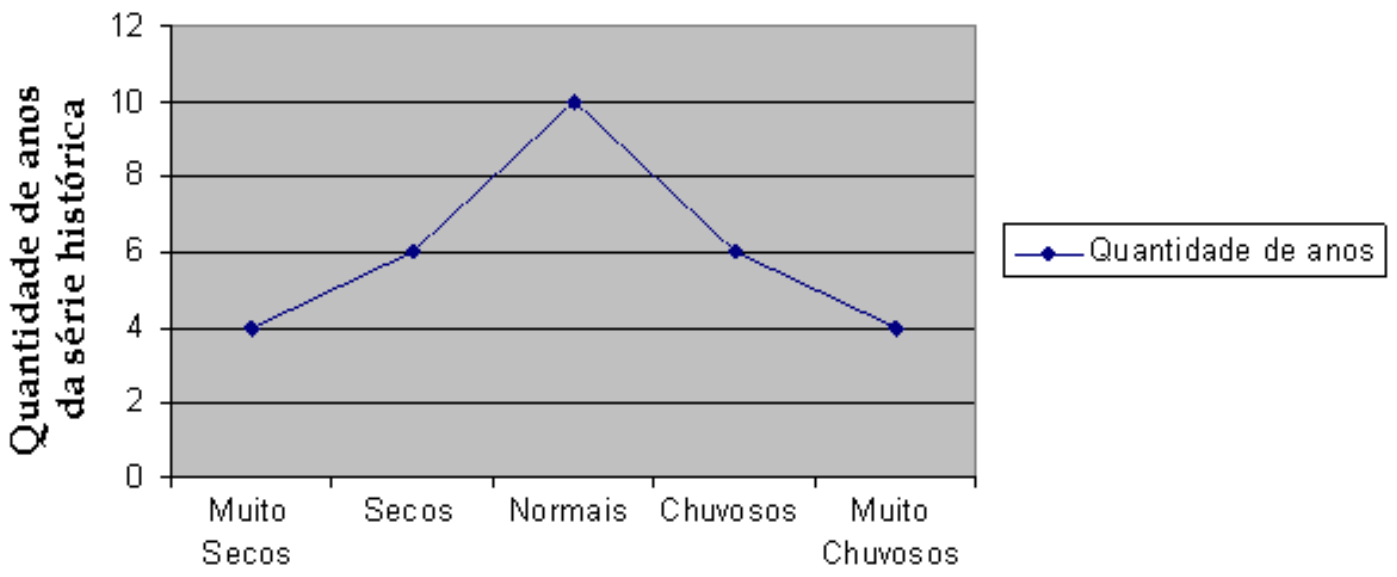

Classificação dos anos

Grafico 1 - Distribuição dos anos secos, normal e chuvoso (1980-2009) no baixo curso do rio Apodi-Mossoró. Fonte: dados elaborados para esta pesquisa.

A análise quantitativa e qualitativa da região indicou que o clima assume importante papel na produção do espaço, pois a partir do conhecimento da dinâmica climática e de sua gênese, podem-se minimizar os efeitos negativos às atividades humanas e direcionar este conhecimento aproveitando a variabilidade temporal para o desenvolvimento socioeconômico. 


\section{CONSIDERAÇÕES FINAIS}

Esta pesquisa não esgota futuras análises. Até o presente (2011) e de acordo com as pesquisas em livros e periódicos, a técnica quantílica ainda não havia sido aplicada na área em estudo, o que mostra a importância científica da pesquisa com a utilização de valores da série histórica distribuídos em cinco classes, segundo os padrões estatísticos.

A utilização da técnica dos quantis demonstrou para o baixo curso a importância teórica e metodológica para o estudo da climatologia geográfica na caracterização de anos secos e chuvosos em uma série histórica de 30 anos (1980-2009) e suas correlações com o padrão de precipitação predominante, com os sistemas atmosféricos e oceânicos e sua atuação na geração de chuva, bem como análise quantitativa e qualitativa para o baixo curso do rio Apodi-Mossoró, contribuindo para o planejamento regional e urbano e possível subsídio para outros trabalhos e projetos de pesquisa.

Sabe-se que os principais aspectos físicos ambientais do Nordeste semiárido brasileiro são: Evaporação elevada, variabilidade espacial e temporal da pluviometria muito acentuada, ocorrência de secas quase periódicas, predomínio de solos cristalinos de baixa profundidade e reduzida capacidade de retenção de água e, em algumas subáreas, avançado processo de desertificação.

$\mathrm{Na}$ área da pesquisa, a correlação destes aspectos podem ser relacionados com a distribuição da vegetação, com o padrão de drenagem, com o regime térmico e hídrico. Essas informações são extremamente úteis na tomada de decisões com relação a algumas práticas agrícolas, entre elas o preparo e o manejo do solo, a semeadura, a colheita, o planejamento do recurso hídrico e o agrometeorológico. Em relação ao número de anos secos, emitindo respostas tanto da erosão na faixa de praia quanto do comportamento do campo de dunas móveis.

Tal metodologia acaba indicando melhor prognóstico para extremos climáticos no semiárido, pois leva em consideração as limitações do clima da região, indicando de forma precisa os valores extremos de chuva para a área de estudo. Afinal, se a chuva acumulada no ano ultrapassar $1200 \mathrm{~mm}$ em uma região como a que foi analisada neste trabalho (de clima semiárido), indicaríamos certamente que este ano pode ser considerado muito chuvoso, enquanto que os mesmos 1200 mm em uma região litorânea podem representar apenas um ano normal. 
Nesse sentido, a classificação dos anos estudados na série histórica e a análise dos anos posteriores através da metodologia proposta neste trabalho (a mesma pode ser utilizada de forma probabilística), podem indicar um possível sinal de alerta para a região, tanto para a possível ocorrência de uma estiagem severa, como para uma possível inundação.

Não se trata apenas de uma observação de um fenômeno natural (a chuva), mas de observála frente a uma vulnerabilidade recorrente na região semiárida do nordeste brasileiro. Tal vulnerabilidade também não está apenas associada às condições climáticas da região, pois sabemos que a vulnerabilidade no semiárido também está relacionada a problemas de infraestrutura local (que não conseguem suportar positivamente à ocorrência de um evento extremo), apresentando indivíduos, famílias ou comunidades com maior exposição (situação de risco) e maior vulnerabilidade (econômica e social) à ocorrência de desastres naturais.

Assim, além de propor uma análise mais confiável de possíveis episódios extremos ocorridos em alguns anos da série histórica analisada ou a probabilidade de ocorrência de eventos extremos nos anos seguintes, a técnica dos quantis permite subsidiar estudos que envolvem uma análise mais profunda da vulnerabilidade da região, principalmente do ponto de vista econômico e social.

Compreender o comportamento da chuva na região e estabelecer sistemas de alerta de estiagens e inundações podem contribuir para uma tomada mais eficaz de medidas que mitiguem os impactos ocasionados pela ocorrência ou não da chuva. Porém, sabemos que a tomada destas medidas fazem parte de um processo um tanto complexo que envolve vários agentes na área em estudo: o indivíduo, a família, a comunidade e o poder público.

Assim, poderemos minimizar os impactos oriundos de eventos extremos na região. Afinal, sabemos que evitar a ocorrência de desastres naturais é humanamente impossível. Porém, é possível criar uma cultura de convivência com o desastre natural, diminuindo consideravelmente os efeitos provenientes do mesmo.

\section{Agradecimentos}

Ao senhor Gilmar Bristot meteorologista da EMPARN por ceder os dados de chuva para a pesquisa. 


\section{REFERÊNCIAS BIBLIOGRÁFICAS}

AGUIAR, F. G. de. À margem da meteorologia do NE. Mossoró: Fundação Guimarães Duque, 1988. (Coleção Mossoroense - série B, n. 487).

AMORIM, M. C. de C. T; MONTEIRO, A. Episódios extremos de precipitação e fragilidade dos ambientes urbanos: exemplos de Portugal e do Brasil. Revista Territorium, v. 17, p. 5-15, 2010.

BAPTISTA, G. M. de M; CARVALHO, J. M; CAMACHO, R. G. V; BIAS, E. de S; ZARA, L. F. Variação sazonal da vegetação e da temperatura de superfície em Mossoró, RN, por meio de dados ASTER. In: SIMPÓSIO BRASILEIRO DE SENSORIAMENTO REMOTO, 13., Goiânia. Anais... Goiânia: INPE, 2005. p. 16-21

BENISTON STEPHENSON. "Extreme Climatic Events and their Evolution under Changing Climatic Conditions", Global and Planetary Change, v. 44, p. 1-9, 2004.

BORGES, J. C. Estudo da variabilidade pluviométrica de Mossoró e região. Mossoró: Fundação Guimarães Duque, 1983. (Coleção Mossoroense, v. 258).

BRANDÃO, A. M. Clima urbano e enchentes na cidade do Rio de janeiro. In: IMPACTOS Ambientais Urbanos no Brasil. Rio de Janeiro: Bertrand Brasil, 2001.

CASTILLO, E., HADI, A.S., BALAKRISHAN, N.; SARABIA, J. M. Extreme Value and Related Models with Applications in Engineering and Science, Wiley Series in Probability and Statistics Hoboken-NJ: Wiley \& Sons, 2005. 362pp.

CPC. Climate Prediction Center. Dados sobre el niño e la niña, desastres naturais em várias parte do mundo. Disponível em: <http://www.cpc.ncep.noaa.gov/>. Acesso em: 12. dez.. 2011.

CPTEC. Centro de Previsão de Tempo e Estudos Climáticos. Dados sobre el niño e la niña. Disponível em: <http://www1.cptec.inpe.br/buscasite.shtml\#la nina>. Acesso em: 12 dez. 2011.

CRUZ, J. da. Ecología social de los desastres. Montevideo: Coscoroba, 2003. 
CARMO FILHO, F.; ESPÍNOLA SOBRINHO, J.; MAIA NETO, J. MOREIRA. Dados Meteorológicos de Mossoró (Janeiro de 1898 a Dezembro de 1990). Mossoró: ESAM, 1991. (Coleção Mossoroense. Série "C" v. 630).

GTI. Cartilha sobre a nova delimitação do semiárido. Disponível em: <http://www.mi.gov.br/desenvolvimentoregional/publicacoes/delimitacao.asp >. Acesso em: 12 dez. 2011.

IDEMA. Instituto de Desenvolvimento Sustentável e Meio Ambiente. Informativo municipal: Mossoró, v.5, p.1-14, 1999. Disponível em: <http//www.idema.rn.gov.br>. Acesso em: 12 dez. 2011.

INMET. Nacional de Meteorologia. Dados sobre precipitação e cadernos explicativos sobre variabilidade climática. Disponível em: <http://www.inmet.gov.br/>. Acesso em 12 dez. 2011.

MOSSORÓ a Nova Cheia. Dados de eventos extremos. Jornal Gazeta do Oeste. Mossoró, 7 de maio de 1985.

MONTEIRO, J. B. Chover, mas chover de mansinho: desastres naturais e chuvas extremas no Estado do Ceará. 2011. Ceará: UEC, 2011.199 p. Dissertação (Mestrado) - Programa de PósGraduação em Geografia, Universidade Estadual do Ceará, Ceará, 2011.

PINKAYAN, S. Conditional probabilities of ocurrence of Wet and Dry Years Over a Large Continental Area. Colorado: State University, Boulder-Co, 1966. (Hidrology papers, n. 12).

ROCHA, A. B. Caracterização climática do Estuário do rio Apodi-Mossoró-RN. Artigo. In: SIMPÓSIO DE CLIMATOlOGIA GEOGRÁFICA, 9., 2010. Fortaleza. Climatologia e Gestão do Território - 26 a 30 de setembro de 2010. Anais... Fortaleza, 2010.

ROCHA, A. B. da. Análise Multitemporal da dinâmica do uso e ocupação do baixo curso do rio Apodi-Mossoró-RN (1989-2009), 2011. 113p. Dissertação (Mestrado) - Programa de PósGraduação em Geografia, Universidade Estadual do Ceará, Ceará, 2011.

XAVIER, T. de M. B. S. Tempo de chuva: estudos climáticos e de previsão para o Ceará e Nordeste setentrional. Fortaleza: ABC Editora, 2001. 
XAVIER, T. de M. B. S.; XAVIER, A. F. S. Papel da componente meridional do vento na costa do Nordeste para a previsão da chuva no Estado do Ceará. In: SIMPÓSIO BRASILEIRO DE RECURSOS HÍDRICOS, 11., 1997, Vitória, ES. Anais... Vitória, 2004. p. 537-543.

XAVIER, T. de M. B. S; XAVIER, A. F. S; ALVES, J. M. B. Quantis e eventos extremos: aplicações em ciências da terra e ambientais. Fortaleza: RDS, 2007. 278 p.

ZANELLA, M. E. Eventos pluviométricos intensos em ambiente urbano: Fortaleza, episódio do dia 29/01/2004. In: SILVA, José Borzachiello da; DANTAS, Eustógio Wanderley Correia; ZANELLA, Maria Elisa; MEIRELES, Antônio Jeovah de Andrade (Org.). Litoral e Sertão: natureza e sociedade no nordeste brasileiro. Fortaleza: Expresso Gráfica, 2006. p 195-207

Artigo recebido em 18/01/2012.

Artigo aceito em 27/02/2012. 